\title{
Perturbation theory for the free energy of classical two-dimensional solids with repulsive inverse-power interactions
}

\author{
Horacio M. Cataldo* and Carlos F. Tejero \\ Facultad de Ciencias Físicas, Universidad Complutense de Madrid, E-28040 Madrid, Spain
}

(Received 23 July 1993; revised manuscript received 1 February 1994)

\begin{abstract}
The free energy of classical two-dimensional solids with repulsive inverse-power interactions is obtained from a recently introduced perturbation theory. We obtain very accurate results for the thermodynamics of the solid phase as compared to the available simulation data.
\end{abstract}

In recent years, the generalized effective liquid approximation (GELA) has been applied with considerable success to the freezing of hard spheres ${ }^{1}$ and hard disks. $^{2}$ When applied to soft interactions the GELA fails and perturbation schemes have been developed to determine the thermodynamics of the solid phase. For three-dimensional solids, a simple perturbation theory around a hard-sphere crystal has been recently applied with good results to the freezing of soft spheres and Yukawa systems. ${ }^{3}$ The theory, which is similar to the perturbation expansion currently used in simple fluids, yields the free energy of the soft-sphere crystal and requires as input the thermodynamics of the hard-sphere crystal. The aim of this report is to check the applicability of this perturbation theory to the study of classical two-dimensional (2D) solids with repulsive inverse-power interactions. We show that a slight modification of the perturbation theory gives very accurate solid free energies for repulsive soft disks. From the free energy of the $2 \mathrm{D}$ solid so obtained we then determine the fluid-solid coexistence by using the available simulation results for the fluid phase free energy.

We therefore consider particles of a classical $2 \mathrm{D}$ solid interacting with an inverse-power pair potential $V(r)=$ $V_{0}(r)+W(r)$ which has been split into a steep shortranged reference part $V_{0}(r)$ and a weak long-ranged perturbation $W(r)$. Following Kang et al. ${ }^{4}$ we introduce a density-dependent potential splitting which shrinks the range of the reference potential to the nearest-neighbor distance of the triangular lattice $\xi$, i.e., $V_{0}(r)=\Theta(\xi-$ $r)\left[V(r)-V(\xi)-(r-\xi) V^{\prime}(\xi)\right]$, with $\Theta(\xi-r)$ the Heaviside step function, $\xi=(2 / \sqrt{3} \rho)^{1 / 2}$, and $\rho$ the average solid density. Let $f$ denote the free energy per particle of the solid phase. After Lutsko and Baus, ${ }^{3}$ by expanding $f$ around the free energy per particle of the reference system $f_{0}$ we find

$$
f=f_{\mathrm{HD}}(\eta)+\frac{1}{2} \sum_{r_{j}>d} W\left(r_{j}\right),
$$

where $f_{0}$ has been further approximated by the free energy per particle of a reference hard-disk solid $f_{\mathrm{HD}}(\eta)$. In Eq. (1) $\eta=\pi \rho d^{2} / 4$ is the packing fraction, $r_{j}$ is the distance of the $j$ th lattice site to the site at the origin, the sum in Eq. (1) running over the Bravais vectors of the triangular lattice, and $d$ is the Barker-Henderson diameter

$$
d=\xi-\int_{0}^{\infty} d r \exp \left[-\beta V_{0}(r)\right]
$$

with $\beta$ the inverse temperature. As it stands, Eq. (1) only involves a lattice sum for the weak long-ranged part of the pair potential, $W(r)$, and the free energy of the hard-disk solid. For the latter we use the results for the $2 \mathrm{D}$ solid obtained from the differential formulation of the GELA (Ref. 2) which are given in Table I. To test the results for the reference hard-disk solid we gather in Table II the fluid-solid coexistence data obtained from the GELA compared to the simulation results. As explained elsewhere, ${ }^{2}$ the quality of the theoretical fluid-solid coexistence data is strongly influenced by the quality of the equation of state of the fluid phase, which has been determined here using the simulation data of Erpenbeck and Luban $^{5}$ and Zollweg and Chester. ${ }^{6}$ It is seen that, while the solid density at coexistence is similar to the result of Zollweg and Chester, ${ }^{6}$ the fluid density at coexistence and the tie-line pressure agree with the older molecular dynamics (MD) results of Alder and Wainwright. ${ }^{7}$ The fractional density change $(2.8 \%)$ is smaller than the results of Lee and Strandburg ${ }^{8}(4.4 \%)$ and Hoover and $\operatorname{Ree}^{9}(3.6 \%)$, but the transition is not so weakly first order as the one found by Zollweg and Chester (1.9\%). From Table I it can be easily found that the pressure of the solid phase obtained from the GELA is understimated with respect to the simulations at $\rho_{S} \sigma^{2} \simeq 0.9{ }^{6}$ whereas it is overestimated at higher densities. ${ }^{10}$

TABLE I. Free energy per particle $\phi_{\mathrm{HD}}(\eta)$, as obtained from the GELA (Ref. 2), for the triangular hard-disk solid as a function of the packing fraction $\eta=\pi \rho \sigma^{2} / 4$, with $\rho$ the density and $\sigma$ the hard-disk diameter. The free energy per particle in Eq. (1) is given by $\beta f_{\mathrm{HD}}(\eta)=\beta \phi_{\mathrm{HD}}(\eta)+2 \ln (\Lambda / \sigma)-1$, $\Lambda$ being the thermal de Broglie wavelength.

\begin{tabular}{cccc}
\hline \hline$\eta$ & $\beta \phi_{\mathrm{HD}}$ & $\eta$ & $\beta \phi_{\mathrm{HD}}$ \\
\hline 0.69 & 3.383 & 0.76 & 4.420 \\
0.70 & 3.521 & 0.77 & 4.592 \\
0.71 & 3.660 & 0.78 & 4.774 \\
0.72 & 3.802 & 0.79 & 4.968 \\
0.73 & 3.948 & 0.80 & 5.176 \\
0.74 & 4.099 & 0.81 & 5.400 \\
0.75 & 4.256 & 0.82 & 5.646 \\
\hline
\end{tabular}


TABLE II. Solid-fluid coexistence data for hard disks as computed from the GELA and compared to the simulation results. Here $\rho_{F}$ and $\rho_{S}$ denote the coexisting densities of the fluid and solid phases, $\sigma$ is the hard-disk diameter, and $\beta P / \rho_{\mathrm{CP}}$ the dimensionless pressure at coexistence, with $\rho_{\mathrm{CP}}$ the close-packing density and $\beta$ the inverse temperature.

\begin{tabular}{lllc}
\hline \hline & $\rho_{F} \sigma^{2}$ & $\rho_{S} \sigma^{2}$ & $\beta P / \rho_{\mathrm{CP}}$ \\
\hline $\mathrm{MD}^{\mathrm{a}}$ & 0.879 & 0.921 & 7.72 \\
$\mathrm{MC}^{\mathrm{b}}$ & 0.880 & 0.912 & 8.08 \\
$\mathrm{MC}^{\mathrm{c}}$ & 0.888 & 0.927 & 8.04 \\
$\mathrm{MC}^{\mathrm{d}}$ & 0.887 & 0.904 & 7.98 \\
$\mathrm{GELA}^{\mathrm{e}}$ & 0.874 & 0.899 & 7.65 \\
\hline \hline
\end{tabular}

${ }^{a}$ Reference 7 .

${ }^{\mathrm{b}}$ Reference 9.

${ }^{\mathrm{c}}$ Reference 8 .

${ }^{\mathrm{d}}$ Reference 6 .

${ }^{\mathrm{e}}$ Reference 2 .

In Eq. (1), the restriction $r_{j}>d$ results from imposing a correlation hole for particles at relative separations less than the Barker-Henderson diameter. ${ }^{3}$ For the triangular lattice $\left(d<\xi=r_{1}\right), W\left(r_{j}\right)=V\left(r_{j}\right)(j \geq 1)$ and the sum in Eq. (1) reduces to the potential energy per particle of the lattice, which can be determined by Ewald summations. We have found that the solid free energies obtained in this way underestimate the simulation results, the small free-energy differences encountered between theory and simulation having a large effect on the location of the solid-fluid coexistence. To understand this systematic behavior we note that the main approximation used to derive Eq. (1) is the replacement of the solid pair correlation function by the step function $\Theta\left(r_{j}-d\right) .^{3}$ This approximation, which ignores the solid structure, yields the result that all the lattice shells are on equal footing in Eq. (1) in spite of the strong correlations at short distances, the most important contribution corresponding to the first nearest-neighbor shell. In view of the extremely simple form of Eq. (1) and of the scarce information about the solid pair distribution function, we here propose (to approximate $f$ to the simulation results) to maintain the step function structure of Lutsko and Baus ${ }^{3}$ but to shift the range of the reference potential to a distance $\xi^{\prime}$ between the first and second nearestneighbor shells, i.e., $\xi^{\prime}=\lambda \xi$ with $1<\lambda<\sqrt{3}$. This approximation yields $W\left(r_{1}\right)=V\left(\lambda r_{1}\right)+r_{1}(1-\lambda) V^{\prime}\left(\lambda r_{1}\right)$ and $W\left(r_{j}\right)=V\left(r_{j}\right)(j \geq 2)$, the first shell giving a different contribution to the lattice sum [Eq. (1)] than the remaining shells. For repulsive inverse-power potentials, the shift of the break point potential lowers the firstshell contribution in Eq. (1) with respect to the original range, while the hard-disk contribution $f_{\mathrm{HD}}(\eta)$ increases due to the increase of the Barker-Henderson diameter [Eq. (2)]. As $f_{\mathrm{HD}}(\eta)$ increases faster than $W\left(r_{1}\right)$ decreases, $f$ draws nearer the simulation result by increasing $\lambda$. We have found that a very small shift, i.e., $\lambda=(2 / \sqrt{3})^{1 / 8} \simeq 1.018$, gives very accurate solid free energies for repulsive inverse-power interactions in the different density regions of interest. We will henceforth consider $\lambda=(2 / \sqrt{3})^{1 / 8}$ as an empirical input in the theory and study its implications.

We first consider two short-ranged inverse-power po-

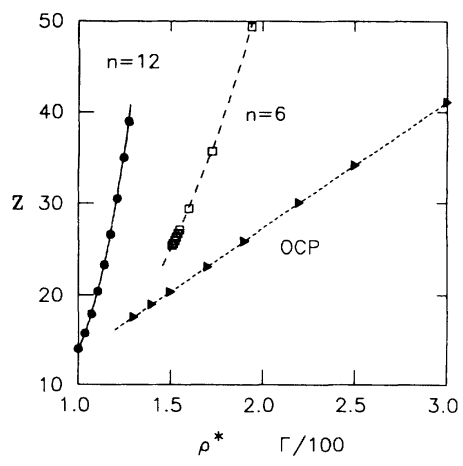

FIG. 1. Compressibility factor $Z=\beta P / \rho$ of the inverse twelfth-power potential (continuous line) and the inverse sixth-power potential (medium-dashed line) vs the temperature-scaled density $\rho^{*}=(\beta \epsilon)^{2 / n} \rho \sigma^{2}$ and compared with the MD simulations of Broughton et al. (Ref. 11) (solid dots) and the MC simulations of Allen et al. (Ref. 12) (open squares). Compressibility factor of the OCP (short-dashed line) vs the coupling parameter $\Gamma=\beta(\pi \rho)^{1 / 2} e^{2}$ (in the plot $-Z / 4$ vs $\Gamma / 100)$ and compared with the MC simulations of Gann et al. (Ref. 13) (solid triangles).

tentials $V(r)=\epsilon(\sigma / r)^{n}(n=12,6)$, with $\epsilon$ and $\sigma$ fixing the energy and length scales, respectively. For both systems the thermodynamic properties may be expressed in terms of a temperature-scaled density $\rho^{*}=(\beta \epsilon)^{2 / n} \rho \sigma^{2}$. In Fig. 1 we plot the compressibility factor of the solid phase $Z=\beta P / \rho, P$ being the pressure, versus $\rho^{*}$ for $n=$ 12 and $n=6$ as computed from Eq. (1) and compared to the MD results of Broughton et al. ${ }^{11}$ and the Monte Carlo (MC) data of Allen et al. ${ }^{12}$ For the one-component plasma (OCP) of ions of charge $e, V(r)=e^{2} / r$, the system has to be stabilized by a continuous background. The thermodynamic quantities depend in this case on the coupling parameter $\Gamma=\beta(\pi \rho)^{1 / 2} e^{2}$. In Fig. 1 we also show the compressibility factor of the OCP versus $\Gamma$ as computed from Eq. (1) together with the MC data of Gann et al. ${ }^{13}$ Notice that our theoretical predictions compare extremely well to the simulations in all the three cases, their accuracy being apparent in Table III, where the solid free energy and the compressibility factor are compared with the simulation data for the two extreme cases $n=12,{ }^{11}$ and $n=1 .^{13}$

TABLE III. Excess free energy per particle $f_{\text {ex }}$ and compressibility factor $Z$ of the classical 2D solid interacting by a repulsive inverse-power potential ( $n=12$ and $n=1)$ compared with the simulation results taken from Broughton et al. (Ref. 11) and Gann et al. (Ref. 13) (in parentheses).

\begin{tabular}{cccc}
\hline \hline$n=12$ & $\rho^{*}$ & $\beta f_{\text {ex }}$ & $Z$ \\
\hline & 1.001 & $5.35(5.35)$ & $13.93(13.99)$ \\
& 1.175 & $8.29(8.29)$ & $26.86(26.62)$ \\
& 1.280 & $11.05(11.01)$ & $40.68(40.20)$ \\
\hline$n=1$ & $\Gamma$ & $\beta f_{\text {ex }}$ & $Z$ \\
\hline & 130 & $-139.41(-139.37)$ & $-70.31(-70.37)$ \\
& 190 & $-205.34(-205.34)$ & $-103.49(-103.56)$ \\
& 300 & $-326.47(-326.54)$ & $-164.32(-164.40)$ \\
\hline \hline
\end{tabular}


TABLE IV. Solid-fluid coexistence data for a classical 2D solid interacting by an inverse twelfth-(sixth-) power potential compared to the simulation results (in parentheses) of Broughton et al. (Ref. 11) and Allen et al. (Ref. 12). Here $\rho_{F}^{*}$ and $\rho_{S}^{*}$ denote the scaled density-temperature variable of the fluid and solid phases, $P^{*}=(\beta \epsilon)^{2 / n+1}\left(P \sigma^{2} / \epsilon\right)$ is the dimensionless pressure at coexistence, and $\Delta s$ is the entropy change per particle at coexistence in units of $k_{B}$.

\begin{tabular}{ccccc}
\hline \hline$n$ & $\rho_{F}^{*}$ & $\rho_{S}^{*}$ & $P^{*}$ & $\Delta s$ \\
\hline 12 & $0.986(0.991)$ & $1.007(1.011)$ & $14.33(14.64)$ & $0.35(0.34)$ \\
6 & $1.506(1.508)$ & $1.519(1.519)$ & $39.27(39.15)$ & $0.30(0.25)$ \\
\hline
\end{tabular}

We have also located the solid-liquid transitions using our theoretical results for the solid phase and the simulation data for the fluid phase. For the inverse twelfthpower potential we have used the eighth-order polynomials of Broughton et al. ${ }^{11}$ to fit the pressure and the chemical potential of the fluid phase in terms of the scaled density-temperature variable $\rho^{*}$ [Eqs. (4.1) and (4.3) in Ref. 11]. For the inverse sixth-power potential we have fitted the reduced excess internal energy of Allen et $a l .{ }^{12}$ (see Table I and Fig. 4 in Ref. 12) in the low$\rho^{*}$ region (up to $\rho^{*}=1.4302$, i.e., without considering the supercooled fluid configurations) with a sixth-order polynomial in $\rho^{*}$. The free energy of the fluid phase has been determined by adding the static lattice contribution and the ideal gas term to the excess part which has been obtained from the excess internal energy by analytical integration. Finally, the excess free energy of the fluid phase has been determined for the OCP by a fit (fourth-order polynomial) in $\Gamma$ of the simulation results of Gann et al. ${ }^{13}$ for coupling parameters $50 \leq \Gamma \leq 120$ (see Table II in Ref. 13). In Table IV we show the coexisting fluid and solid densities, the pressure at coexistence, and the entropy change per particle for the inverse-power potentials $n=12$ and $n=6$ obtained from our theoretical calculations and compared to the simulation results. In both cases a fair agreement is found, the quality of the theoretical fluid-solid coexistence results being fairly sensitive to the fit used for the fluid phase. We separately consider the OCP fluid-solid transition. In a firstprinciples study Weeks, ${ }^{14}$ using the scaling property for inverse-power-law potentials and the Clausius-Clapeyron equation, found no density change on melting for long- ranged forces in a uniform neutralizing background. The transition is therefore determined by the crossing of the solid and fluid free energies. Our theoretical calculations lead to a very weak first-order transtion $\left(\Gamma_{F}=100.521\right.$; $\left.\Gamma_{S}=100.475\right)$ with an extremely small fractional density change $\left(\simeq 4.5 \times 10^{-4}\right)$ and entropy change at coexistence $\Delta s / k_{B}=0.15$. We notice, however, that because of the small free-energy differences involved between the solid and fluid phases (for $50<\Gamma<150$ ), it is hazardous to assess the crossing of the two curves. Experimental studies show that the transition for a $2 \mathrm{D}$ sheet of electrons occurs at $\Gamma=137 \pm 15,{ }^{15}$ while $\mathrm{MC}$ and $\mathrm{MD}$ simulations yield $\Gamma=95 \pm 2,{ }^{16} \Gamma=118-130,{ }^{17}$ and $\Gamma=125 \pm 15 .{ }^{13}$ Systematic studies of size dependence and much longer runs are necessary to understand these large discrepancies.

To sum up, we have applied a very simple hard-disk perturbation theory to the study of classical 2D solids with repulsive inverse-power interactions. Our estimates for the free energy of the solid phase are in good agreement compared to the simulation results for different inverse-power interactions. The solid-fluid transition is accurately described for short-ranged potentials, the major differences with respect to the simulations being found for the OCP.

We are very grateful to $M$. Baus for helpful comments. H.M.C. acknowledges financial support from the Dirección General de Investigación Científica y Técnica (Spain) (SB92-A07794241). This work has been supported by the DGICYT (PB91-0378).
* Permanent address: Departamento de Física, Facultad de Ciencias Exactas y Naturales, Universidad de Buenos Aires, 1428, Buenos Aires, Argentina.

${ }^{1}$ J. F. Lutsko and M. Baus, Phys. Rev. Lett. 64, 761 (1990); Phys. Rev. A 41, 6647 (1990).

${ }^{2}$ C. F. Tejero and J. A. Cuesta, Phys. Rev. E 47, 490 (1993).

${ }^{3}$ J. F. Lutsko and M. Baus, J. Phys. Condens. Matter 3, 6547 (1991); C. F. Tejero, J. F. Lutsko, J. L. Colot, and M. Baus, Phys. Rev. A 46, 3373 (1992).

${ }^{4}$ H. S. Kang, T. Ree, and F. H. Ree, J. Chem. Phys. 84, 4547 (1986).

${ }^{5}$ J. J. Erpenbeck and M. Luban, Phys. Rev. A 32, 2920 (1985).

${ }^{6}$ J. A. Zollweg and G. V. Chester, Phys. Rev. B 46, 11186 (1992).

${ }^{7}$ B. J. Alder and T. E. Wainwright, Phys. Rev. 127, 359 (1962).

${ }^{8}$ J. Lee and K. J. Strandburg, Phys. Rev. B 46, 11190
(1992).

${ }^{9}$ W. G. Hoover and F. H. Ree, J. Chem. Phys. 49, 3609 (1968).

10 J. A. Zollweg, G. V. Chester, and P. W. Leung, Phys. Rev. B 39, 9518 (1989).

${ }^{11}$ J. Q. Broughton, G. H. Gilmer, and J. D. Weeks, Phys. Rev. B 25, 4651 (1982).

${ }^{12}$ M. P. Allen, D. Frenkel, W. Cignac, and J. P. McTague, J. Chem. Phys. 78, 4206 (1983).

${ }^{13}$ R. C. Gann, S. Chakravarty, and G. V. Chester, Phys. Rev. B 20, 326 (1979).

${ }^{14}$ J. D. Weeks, Phys. Rev. B 24, 1530 (1981).

${ }^{15}$ C. C. Grimes and G. Adams, Phys. Rev. Lett. 42, 795 (1979).

${ }^{16}$ R. W. Hockney and T. R. Brown, J. Phys. C 8, 1813 (1975).

${ }^{17}$ R. K. Kalia, P. Vashishta, and S. W. de Leeuw, Phys. Rev. B 23, 4794 (1981). 